\title{
Tracking Radio-tagged Masu Salmon (Oncorhynchus masou) from the Sky by Use of Drone
}

\author{
Arimune Munakata ${ }^{1}$, Masaya Suzuki ${ }^{2}$, and Tomoichiro Tanaka ${ }^{3}$ \\ ${ }^{1}$ Department of Biology, Miyagi University of Education, 149 Aobaku Sendai, Miyagi 980-0845, Japan \\ ${ }^{2}$ Suzuki Giken, 2281-12 Yamaguma, Tachiarai, Fukuoka 830-1226, Japan \\ ${ }^{3}$ Tanaka Sanjiro Co., Ltd. 1562 Ogori, Ogori, Fukuoka 838-0141, Japan
}

Keywords: bio telemetry, drone, masu salmon, Oncorhynchus masou, radio tag

In order to track the location and movement of salmonids in rivers and lakes, several types of external tags such as fin-clipped, ribbon, spaghetti, elastomer (Choe and Yamazaki 1996), etc. are traditionally used. However, it has been a problem that such tags should be checked visually by recapturing the released fish in various locations, as the tag detection rate largely depends on the sampling locations and procedures. In order to improve the recapture rate, fish collection procedures by use of net, trap, fishing rod, electric fisher, etc. have also been developed.

Recently, several types of remote tags such as Passive Integrated Transponder (PIT), acoustic, and radio tags have been devised. These tags are advantageous because they allow researchers to know the location and movement of experimental fish without recapturing them. In these systems, however, it is necessary to set stationary receivers - including antennas - along rivers or lakeshores and therefore the detection rates fundamentally depend on the number of receivers (antennas) and their reception range (Binder et al. 2017). As another option, the researcher can move along the rivers or lakeshores on foot or in a research vessel with a portable receiver. In a previous river experiment, researchers floated down the river in a research boat (Aarestrup et al. 1999). In our study, we walked up and down along the rivers (Munakata et al. unpublished data). In both cases, it is nearly inevitable to come across several types of barriers such as waterfalls, cliffs, or bushes, etc. In the case of lakes, researchers should move in wider ranges to detect and track experimental fishes.

In order to decrease study effort and improve detection efficiency, we developed a new tracking method for radio-tagged salmonids by using a flying drone. In this system, the biggest concern was to avoid radio disturbances by mechanical noises from the drone, so we developed a new receiver which is composed of a noise filter, amplifier, radio signal analyzer using micro processer, and a memory unit (micro-SD memory card). These parts were connected to a directional antenna and a portable lithium-ion battery and were attached to a drone (Phantom II, DJI Co., Ltd.) (Fig. 1). We used two types of radio tags (MCFT3 and nanotag NTF-3-1, Lotek Co., Ltd.) for which the frequency is $151.89 \mathrm{MHz}$. Based on the specific transmitting patterns, we could discriminate tag identification. During the flight, data receptions (radio strength and ID) were analyzed and recorded on a micro-SD memory card with its location based on GPS data.

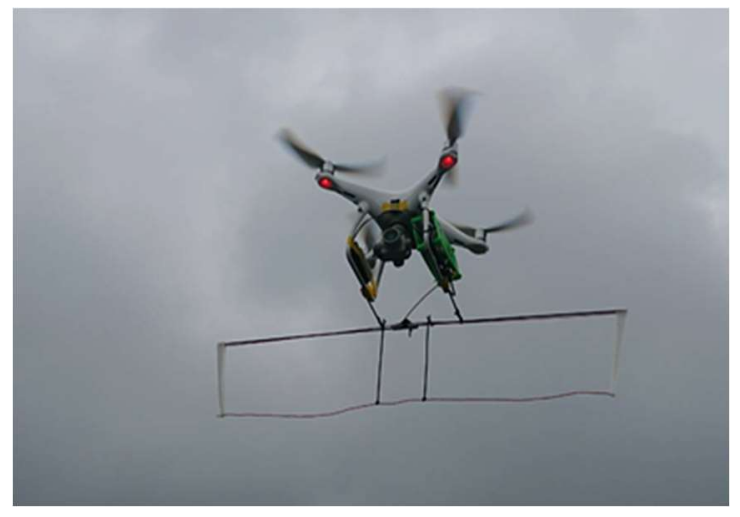

Fig. 1. A photograph of the radio receiving drone. All receivers, including directional antenna, are attached to the drone.

One of the difficulties of flying the drone over small streams is to avoid structures and barriers such as trees, cliffs, bridges, and electrical wires. In the case of lakes, it is also difficult to fly the drone with equal pitches. Thus, in this study, we programmed flight route and altitudes based on tridimensional survey flight data. Basically, we flew the drone at a higher and safer altitude and took still photos over the expected flight areas. Then the flight route and altitude were programmed using metashape (Agisoft LLC) and UgCS (GeoMetrics Inc.) software. 
One of the advantages of radio tracking by a flying drone is to know the detection result in real-time. By doing so, the operation can be finished in the shortest time and with the least effort. To implement real-time radio tracking, we developed an additional system. When the receiver detects a radio tag, an LED lamp attached to the drone flashes (Munakata et al. unpublished data) allowing the operator to grab detection time and total number of radio tags through a camera installed on the drone - the information is then displayed on the remote-control panel. After the flight, detailed tracking data including flight route, tag ID, radio strength, and tag location can be downloaded from the micro-SD memory card.

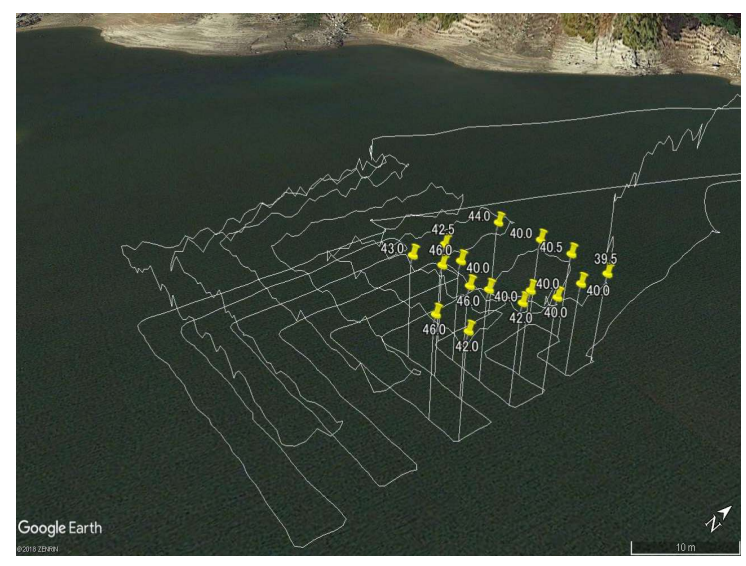

Fig. 2. A sample picture of the calibration test for the radio tag set underwater. Two types of white lines show the programed flight course (lower) and actual flight course (upper). Yellow pins and numbers represent the detection points and radio strength. Smallest number indicates the highest radio strength.

By use of such a receiving system, we calibrated the detection depth and radius for the radio tags in a lake. In the case of Lotek MCFT3 (Frequency $151.89 \mathrm{MHz}$, output $0.016 \mathrm{~mW}$ ), maximum detection depth and radius were approximately $8 \mathrm{~m}$ and $15 \mathrm{~m}$, respectively, when the drone flew at an altitude of $10 \mathrm{~m}$ (Munakata et al. unpublished data). The detection range tended to decrease in relation to the flight altitude, but we were able to mark $4 \mathrm{~m}$ depth and $10 \mathrm{~m}$ radius at an altitude of $30 \mathrm{~m}$. In these trials, moreover, we could fly the drone at a constant pitch and altitude (see detail in Fig. 2).

In 2019, we demonstrated the radio tracking experiment on a Biwa salmon (Oncorhynchus masou subsp.) (Kuwahara and Iguchi 2007). On 9 November, five adult Biwa salmon were caught by set net near the mouth of Ado River flowing into Lake Biwa, Shiga prefecture, Japan. The five individuals were anesthetized, and a radio tag (MCFT3) was implanted into their abdominal cavities through a $1 \mathrm{~cm}$ incision made by a surgical blade. After the wound was stitched, the fish were reared in a plastic cage placed in the river for about one hour, and then the fish were released near the set net. Two hours after the release, and one to five days after the release, we attempted to detect the location of the experimental fish. One day later, we detected all the fish on the upstream side of the river (Munakata et al. unpublished data). Among five individuals, three fish moved approximately one to three kilometers upstream from the release point. On the other hand, radio signals from the other two fish were detected close to the release point so we attempted to visually locate these fish based on the radio signals. These two fish (a female and male) died but these fish seemed to have released a major part of their matured eggs and sperm. Five days after the release, on the other hand, one of three experimental fish was found approximately $7 \mathrm{~km}$ upstream from the release point. Thus, in this experiment, we could detect the location of all five tagged Biwa salmon adults along the river using the drone receiving system. During the experiment, we attached not only the radio tag, but also an external ribbon tag on the dorsal part of the fish. During the radio tracking, however, we could not visually locate the experimental fish except for the two dead fish. From these results, the drone receiving system was considered to be a highly efficient tracking method.

In response to this result, we also attempted to track the movement of Taiwan salmon (Oncorhynchus masou formosanus) (Hsu et al. 2010) in a steep mountain stream in Wuling, Taiwan. In November 2019, five Taiwan salmon which were caught by electro fisher were anesthetized and a radio tag (nano tag NTF-3-1) was implanted into the abdominal cavity of each fish. After the implantation, the fish were reared in a hatchery FRP tank overnight and then released near the sampling point. One and two days after the release, we attempted to detect the location of these fish. One day after the release, we found that two fish moved approximately 200 to $300 \mathrm{~m}$ upstream from the release point while three fish stayed near the release point (Munakata et al. unpublished data). Two days after the release, on the other hand, it was demonstrated that all five fish gathered within $50 \mathrm{~m}$ from the released point. In this case, we also tracked the location of five Taiwan salmon in a small mountain stream. Moreover, it is also noteworthy that we could discriminate tag identification with five experimental fish gathered in the same area. 
Based on these results, it is indicated that the drone receiving system can be a useful tool for tracking the location and movement of radio-tagged salmonid in small streams and shallow lakes.

\section{REFERENCES}

Aarestrup, K., N. Jepsen, G. Rasmussen, and F. Økland. 1999. Movement of two strains of radio tagged Atlantic salmon, Salmo salar L., smolt through a reservoir. Fish. Manage. Ecol. 6: 97-107.

Binder, T.R., S.A. Farha, H.T. Thompson, C.M. Holbrook, R.A. Bergstedt, S.C. Riley, and C.C. Krueger. 2017. Fine-scale acoustic telemetry reveals unexpected lake trout, Salvelinus namaycush, spawning habitats in northern Lake Huron, North America. Ecol. Freshw. Fish. 27: 594-605. doi.org/10.1111/eff.12373.

Choe, M.K., and F. Yamazaki. 1996. An evaluation of fluorescent elastomer tagging method for juvenile masu salmon (Oncorhynchus masou). Fish Genetics and Breeding Science 23: 41-50. (In Japanese)

Kuwahara, M., and K. Iguchi. 2007. Occurrence of summer upstream migration in Biwa salmon (Oncorhynchus masou subsp.). Jpn. J. Ichthyol. 54(1): 15-20. (In Japanese with English abstract)

Hsu, C.B., C.S. Tzeng, C.H. Yeh, W.H. Kuan, M.H. Kuo, and H.J. Lin. 2010. Habitat use by the Formosan landlocked salmon Oncorhynchus masou formosanus. Aquat. Biol. 10: 227-239. doi.org/10.3354/ab00280. 